\title{
ON THE OPTIMAL CONSTANT FOR THE BERGMAN PROJECTION ONTO THE BLOCH SPACE
}

\author{
Antti Perälä \\ University of Helsinki, Department of Mathematics and Statistics \\ P.O. Box 68, FI-00014 Helsinki, Finland; antti.i.perala@helsinki.fi
}

\begin{abstract}
In this note we obtain the best constant for the Bergman projection from $L^{\infty}$ onto the Bloch space. We also consider the question for the projection from $C(\overline{\mathbf{D}})$ onto the little Bloch space.
\end{abstract}

\section{Introduction}

Bergman type projections are central operators when dealing with questions related to analytic function spaces over domains of the complex plane. They are studied in their own right, or in connection to Toeplitz operators, for instance. One often wants to have bounded Bergman projections, while the exact operator norm of the projection is less important. Let $\mathbf{D}=\{|z|<1\}$ be the open unit disk of the complex plane and $d A(z)=\pi^{-1} d x d y$ stand for the normalized Lebesgue area measure. The Bergman projection $P$ is then defined as

$$
\operatorname{Pf}(z)=\int_{\mathbf{D}} \frac{f(w) d A(w)}{(1-z \bar{w})^{2}}, \quad z \in \mathbf{D} .
$$

Let $0<p<\infty$ and consider the Bergman space $A^{p}$ consisting of those analytic functions $f$ on $\mathbf{D}$, satisfying

$$
\int_{\mathbf{D}}|f(z)|^{p} d A(z)<\infty
$$

The space $H^{\infty}$ is the space of bounded analytic functions on D. For $1<p<\infty$ it is known that the Bergman projection maps $L^{p}$ boundedly onto $A^{p}$. The exact operator norm is, however, difficult to verify unless $p=2$, in which case the norm is one. In [1] K. Zhu studied the asymptotic behaviour of the the norm of $P$ when $p \rightarrow 1^{+}$ and $p \rightarrow \infty$. It is known that the Bergman projection is not bounded $L^{1} \rightarrow A^{1}$ or $L^{\infty} \rightarrow H^{\infty}$.

Let us now consider the Bloch space consisting of analytic functions $f: \mathbf{D} \rightarrow \mathbf{C}$, for which

$$
\|f\|_{\mathcal{B}}:=\sup _{z \in \mathbf{D}}\left(1-|z|^{2}\right)\left|f^{\prime}(z)\right|<\infty .
$$

It is known that the Bergman projection $L^{\infty} \rightarrow \mathcal{B}$ is bounded and onto. A good source for theory of Bergman projections and the Bloch space is the book [2]. Note

doi:10.5186/aasfm.2012.3722

2010 Mathematics Subject Classification: Primary 47B35.

Key words: Bergman projection, Bloch space.

The author is supported by The Finnish National Graduate School in Mathematics and its Applications and partially supported by the Academy of Finland project "Functional analysis and applications". 
that $\|\cdot\|_{\mathcal{B}}$ is not a norm, so by boundedness we mean that there exists $C>0$ such that

$$
\|P f\|_{\mathcal{B}} \leq C\|f\|_{\infty}
$$

for every $f \in L^{\infty}$. In this note we calculate the smallest possible $C$ such that (1.1) remains true for every $f \in L^{\infty}$. As a main result of this paper we get that the optimal constant equals $8 / \pi$. The same thing also is shown to hold for $P: C(\overline{\mathbf{D}}) \rightarrow \mathcal{B}_{0}$, where $\mathcal{B}_{0}$ is the little Bloch space.

The author wishes to thank Academy Professor Kari Astala and Professor Kehe Zhu for showing interest in the problem and for the encouragement during the course of work. The author also thanks Juhani Koivisto for his nice piece of advice for the final stage of the proof.

\section{The result}

Lemma 2.1. The optimal constant $C$ is given by

$$
C=2 \sup _{z \in \mathbf{D}} \int_{\mathbf{D}} \frac{\left(1-|z|^{2}\right)|w| d A(w)}{|1-z \bar{w}|^{3}} .
$$

Proof. For $f \in L^{\infty}$ we can differentiate

$$
\operatorname{Pf}(z)=\int_{\mathbf{D}} \frac{f(w) d A(w)}{(1-z \bar{w})^{2}}
$$

under the integral sign to obtain

$$
\|P f\|_{\mathcal{B}}=2 \sup _{z \in \mathbf{D}}\left|\int_{\mathbf{D}} \frac{\left(1-|z|^{2}\right) \bar{w} f(w) d A(w)}{(1-z \bar{w})^{3}}\right| .
$$

If now $\epsilon>0$, there exist a function $g_{\epsilon} \in L^{\infty}$ and $\zeta \in \mathbf{D}$ such that $\left\|g_{\epsilon}\right\|_{\infty} \leq 1$ and

$$
\left(1-|\zeta|^{2}\right)\left|\left(P g_{\epsilon}\right)^{\prime}(\zeta)\right|>C-\epsilon \text {. }
$$

On the other hand, for every $z \in \mathbf{D}$, we can pick

$$
f_{z}(w)=\frac{w|1-z \bar{w}|^{3}}{|w|(1-\bar{z} w)^{3}} .
$$

Note that $\left\|f_{z}\right\|_{\infty}=1$ for every $z \in \mathbf{D}$ and that for each $g \in L^{\infty}$ with $\|g\|_{\infty} \leq 1$ we always have

$$
\left|\int_{\mathbf{D}} \frac{\bar{w} g(w) d A(w)}{(1-z \bar{w})^{3}}\right| \leq\left|\int_{\mathbf{D}} \frac{\bar{w} f_{z}(w) d A(w)}{(1-z \bar{w})^{3}}\right| \leq \int_{\mathbf{D}} \frac{|w| d A(w)}{|1-z \bar{w}|^{3}} .
$$

In particular

$$
\left(1-|\zeta|^{2}\right)\left|\left(P f_{\zeta}\right)^{\prime}(\zeta)\right| \geq\left(1-|\zeta|^{2}\right)\left|\left(P g_{\epsilon}\right)^{\prime}(\zeta)\right|
$$

The claim now follows.

Our next aim is to study integrals of type (2.1). To this end, we denote by $F: \mathbf{D} \rightarrow \mathbf{C}$ the following function:

$$
F(z)=\int_{\mathbf{D}} \frac{\left(1-|z|^{2}\right)|w| d A(w)}{|1-z \bar{w}|^{3}} .
$$


Proposition 2.2. The function $F$ is radial, that is $F(z)=F(|z|)=F(r)$ for every $z=r e^{i \theta} \in \mathbf{D}$.

Proof. Suppose $z=r e^{i \theta}$ and make a change of variables $w \mapsto e^{i \theta} w$. Then

$$
F(z)=\int_{\mathbf{D}} \frac{\left(1-|z|^{2}\right)|w| d A(w)}{|1-z \bar{w}|^{3}}=\int_{\mathbf{D}} \frac{\left(1-r^{2}\right)|w| d A(w)}{|1-r \bar{w}|^{3}}=F(r),
$$

and the claim is proved.

To proceed with our proof, we want to express $F$ in a slightly different form.

Proposition 2.3. For every $z \in \mathbf{D}$, we have

$$
F(z)=\int_{\mathbf{D}} \frac{|z-w| d A(w)}{|1-z \bar{w}|^{2}} .
$$

Proof. We use the Möbius map $\varphi_{z}(w)=(z-w) /(1-\bar{z} w)$ for $z, w \in \mathbf{D}$. It is known that $\varphi_{z}$ is a conformal self-map of the unit disk, $\varphi_{z}$ interchanges $z$ and 0 and that $\varphi_{z}$ is its own inverse under composition. Moreover, the complex Jacobian $J_{z}$ of $\varphi_{z}$ is given by

$$
J_{z}=\frac{\left(1-|z|^{2}\right)^{2}}{|1-z \bar{w}|^{4}}
$$

We first note that

$$
1-z \overline{\varphi_{z}(w)}=\frac{1-|z|^{2}}{1-z \bar{w}}
$$

Using change of variables, we now have

$$
\begin{aligned}
F(z) & =\int_{\mathbf{D}} \frac{\left(1-|z|^{2}\right)|w| d A(w)}{|1-z \bar{w}|^{3}}=\int_{\mathbf{D}}\left|\varphi_{z}(w)\right| \frac{\left|1-z \overline{\varphi_{z}(w)}\right| d A(w)}{1-|z|^{2}} \\
& =\int_{\mathbf{D}} \frac{|z-w| d A(w)}{|1-z \bar{w}|^{2}}
\end{aligned}
$$

which was the claim.

By proposition 2.3, we can deduce that $F$ extends to a continuous function on $\overline{\mathrm{D}}$. Because $F$ is radial, we set

$$
F(1)=\lim _{r \rightarrow 1^{-}} F(r)=\int_{\mathbf{D}} \frac{d A(w)}{|1-w|}
$$

and the continuous extension is well-defined, so we will think of $F$ as a function defined on $\overline{\mathbf{D}}$. We also know that if $F$ obtains its maximum on the boundary, the optimal constant would be $C=2 F(1)$.

Proposition 2.4. The function $F$ obtains its maximum at any point on the boundary of the unit disk.

Proof. For each $w \in \mathbf{D}$ the map $z \mapsto(z-w) /(1-z \bar{w})^{2}$ is analytic. Therefore $G_{w}(z):=|z-w| /|1-z \bar{w}|^{2}$ is subharmonic for every $w \in \mathbf{D}$. Then, for the Laplacian we have

$$
\triangle G_{w}(z) \geq 0
$$


for every $z, w \in \mathbf{D}, z \neq w$. From formula (2.3) it is easily seen that $F$ is $C^{2}$ on $\mathbf{D}$. It now follows that

$$
\triangle F(z)=\triangle \int_{\mathbf{D}} \frac{|z-w| d A(w)}{|1-z \bar{w}|^{2}}=\int_{\mathbf{D} \backslash\{z\}} \triangle G_{w}(z) d A(w) \geq 0,
$$

for every $z \in \mathbf{D}$. Therefore $F$ is subharmonic and the proof is finished by use of the maximum principle.

We are now ready to state and prove the main theorem with ease.

Theorem 2.5. The optimal constant $C$ for the inequality

$$
\|P f\|_{\mathcal{B}} \leq C\|f\|_{\infty}
$$

equals $8 / \pi$.

Proof. By the preceding set of results, the optimal constant is given by

$$
C=2 \int_{\mathbf{D}} \frac{d A(w)}{|1-w|}
$$

so it remains to calculate this integral. By using real variable theory, we can write

$$
\begin{aligned}
\int_{\mathbf{D}} \frac{d A(w)}{|1-w|} & =\pi^{-1} \int_{-1}^{1} \int_{-\sqrt{1-x^{2}}}^{\sqrt{1-x^{2}}} \frac{d y d x}{\sqrt{(1-x)^{2}+y^{2}}} \\
& =\pi^{-1} \int_{-1}^{1}\left[\operatorname{arsinh}\left(\frac{\sqrt{1-x^{2}}}{1-x}\right)-\operatorname{arsinh}\left(\frac{-\sqrt{1-x^{2}}}{1-x}\right)\right] d x \\
& =\pi^{-1} 2\left[x \operatorname{arsinh}\left(\frac{\sqrt{1+x}}{\sqrt{1-x}}\right)+\sqrt{2+2 x}-\operatorname{artanh}\left(\frac{\sqrt{1+x}}{\sqrt{2}}\right)\right]_{-1}^{1} .
\end{aligned}
$$

The last identity is easily verified by taking a derivative (the author obtained the formula by the use of computers). Now, it can be verified by using the logarithmic formulas for arsinh and artanh that

$$
\lim _{x \rightarrow 1^{-}}\left[\operatorname{arsinh}\left(\frac{\sqrt{1+x}}{\sqrt{1-x}}\right)-\operatorname{artanh}\left(\frac{\sqrt{1+x}}{\sqrt{2}}\right)\right]=0 .
$$

The rest of the boundary terms are easily computed and thus we obtain

$$
\int_{\mathbf{D}} \frac{d A(w)}{|1-w|}=4 / \pi
$$

It follows that $C=8 / \pi$.

Let $\mathcal{B}_{0}$ stand for the little Bloch space, consisting of those $f \in \mathcal{B}$ with

$$
\lim _{|z| \rightarrow 1^{-}}\left(1-|z|^{2}\right)\left|f^{\prime}(z)\right|=0 \text {. }
$$

It is known that $P: C(\overline{\mathbf{D}}) \rightarrow \mathcal{B}_{0}$ is bounded. The following corollary deals with the optimal constant for this projection. 
Corollary 2.6. The optimal constant $D$ for the inequality

$$
\|P f\|_{\mathcal{B}} \leq D\|f\|_{\infty}, \quad f \in C(\overline{\mathbf{D}})
$$

equals $8 / \pi$.

Proof. Since $C(\overline{\mathbf{D}}) \subset L^{\infty}$ it is easy to see that $D \leq C$. We want to show that $D \geq C$. The functions $f_{z}$ in $(2.2)$ are not in $C(\overline{\mathbf{D}})$. For $0<\epsilon<1 / 2$ define functions $f_{z}^{\epsilon}$ by

$$
f_{z}^{\epsilon}(w)= \begin{cases}f_{z}(w) & \text { if }|w|>\epsilon \\ f_{z}(w)|w| / \epsilon & \text { if }|w| \leq \epsilon\end{cases}
$$

Clearly $f_{z}^{\epsilon} \in C(\overline{\mathbf{D}})$ so $P f_{z}^{\epsilon} \in \mathcal{B}_{0}$ for every $z \in \mathbf{D}$ and $0<\epsilon<1 / 2$. If now $z \in \mathbf{D}$, then

$$
\begin{aligned}
\left|P\left(f_{z}^{\epsilon}-f_{z}\right)(z)\right| \leq & 2 \int_{|w| \leq \epsilon} \frac{|w|\left(1-|z|^{2}\right)\left|f_{z}^{\epsilon}-f_{z}\right|(w) d A(w)}{|1-z \bar{w}|^{3}} \\
& +2 \int_{|w|>\epsilon} \frac{|w|\left(1-|z|^{2}\right)\left|f_{z}^{\epsilon}-f_{z}\right|(w) d A(w)}{|1-z \bar{w}|^{3}} \\
\leq & 32 \epsilon^{3} .
\end{aligned}
$$

Therefore $P f_{z}^{\epsilon}(z) \rightarrow P f_{z}(z)$ as $\epsilon \rightarrow 0$. From this, it is immediately deduced that the optimal constant of the corollary is the same as in the main theorem: $D=8 / \pi$.

\section{References}

[1] ZHU, K.: A sharp norm estimate of the Bergman projection on $L^{p}$ spaces. - Contemp. Math. 404, 2006, 199-205.

[2] Zhu, K.: Operator theory in function spaces. - Math. Surveys Monogr. 138, 2nd edition, Amer. Math. Soc., Providence, RI, 2007.

Received 24 May 2011 